\title{
ON KILMISTER'S CONDITIONS FOR THE EXISTENCE OF LINEAR INTEGRALS OF DYNAMICAL SYSTEMS
}

\author{
by R. H. BOYER \\ (Received 17th March 1964)
}

Kilmister (1) has considered dynamical systems specified by coordinates $q^{\alpha}(\alpha=1,2, \ldots, n)$ and a Lagrangian

$$
L=\frac{1}{2} a_{\alpha \beta} \dot{q}^{\alpha} \dot{q}^{\beta}+a_{\alpha} \dot{q}^{\alpha}+a
$$

(with summation convention). He sought to determine generally covariant conditions for the existence of a first integral, $b_{\alpha} \dot{q}^{\alpha}=$ constant, linear in the velocities. He showed that it is not, as is usually stated, necessary that there must exist an ignorable coordinate (equivalently, that $b_{\alpha}$ must be a Killing field:

$$
b_{\alpha ; \beta}+b_{\beta ; \alpha}=0
$$

where covariant derivation is with respect to $a_{\alpha \beta}$ ). On the contrary, a singular integral, in the sense that $b_{a} \dot{q}^{\alpha}=1$ for all time if satisfied initially, need not be accompanied by an ignorable coordinate.

In fact, Kilmister has shown that necessary and sufficient conditions for the existence of a linear first integral are

$$
\begin{aligned}
b_{\alpha ; \beta}+b_{\beta ; \alpha} & =2 \theta b_{\alpha} b_{\beta}, \\
b^{\rho}\left(a_{\rho ; \beta}-a_{\beta ; \rho}\right) & =\phi b_{\beta}, \quad \ldots \\
b^{\rho} a_{, \rho} & =\phi-\theta .
\end{aligned}
$$

In this note, a simple consequence of these equations will be exploited. Namely, since the kinetic energy matrix $a_{\alpha \beta}$ must be positive definite, $b_{\alpha} b^{\alpha} \neq 0$, so that, from (2), $\phi=0$. Moreover, from (1)

$$
b_{a ; \beta} b^{\alpha} b^{\beta}=\theta\left(b_{\alpha} b^{\alpha}\right)^{2} \text {, or } \theta=\left(-1 / 2 b_{\alpha} b^{\alpha}\right)_{, \beta} b^{\beta} .
$$

Finally, (3) says $\left(a-1 / 2 b_{\alpha} b^{\alpha}\right)_{\beta} b^{\beta}=0$.

For $n=2$ these equations can be integrated completely. Choosing a coordinate system $(x, y)$ for which $b^{\alpha}=(1,0), b_{\alpha}=a_{x 1}$, and (1) becomes

$$
a_{\alpha \beta, 1}=a_{11,1} a_{\alpha 1} a_{\beta 1} / a_{11}^{2},
$$

which is satisfied identically for $\alpha=\beta=1$ and for $\alpha=1, \beta=2$ gives

$$
a_{12}=a_{11} A(y) \text {. }
$$

For $\alpha=\beta=2$ we find $a_{22}=a_{11} A^{2}(y)+B^{2}(y) . \quad A(y)$ and $B(y)$ are arbitrary. 
(2) becomes $a_{1,2}=a_{2,1}$ or $a_{\alpha}=V_{, \alpha}$, say. But since such terms will not contribute to the equations of motion, they may be dropped from the Lagrangian. (3) requires $a=\frac{1}{2 a_{11}}+C(y)$. Thus

$$
L=\frac{1}{2}\left[a_{11} \dot{x}^{2}+2 a_{11} A(y) \dot{x} \dot{y}+a_{11} A^{2}(y) \dot{y}^{2}+B^{2}(y) \dot{y}^{2}\right]+\frac{1}{2 a_{11}}+C(y) .
$$

Let $\xi=x+\int A(y) d y$ and $\eta=\int B(y) d y$. Then

$$
L=\frac{1}{2}\left[a_{11} \dot{\xi}^{2}+\dot{\eta}^{2}\right]+\frac{1}{2 a_{11}}+D(\eta)
$$

$a_{11}$ is an arbitrary positive function of $\xi$ and $\eta$. This Lagrangian is mentioned by Kilmister, who implied it to be of less general interest. The integration procedure used here fails when $n>2$.

The equation (1) has been noticed by Rayner (2) while studying rigid motions in general relativity. There, of course, $a_{\alpha}$ and $a$ have no analogues. Kilmister's result corresponds to the fact that if a motion $b^{\alpha}$ satisfies (1) (implying that the motion is rigid) and if $u^{\alpha}$ is a unit tangent to a geodesic, then $b_{\alpha} u^{\alpha}=0$ along the entire geodesic if it is satisfied at one point.

\section{REFERENCES}

(1) C. W. Kilmister, Proc. Edinburgh Math. Soc. (II), 12 (1961), 13 (Mathematical Note).

(2) C. B. Rayner, C. R. Acad. Sci. Paris, 249 (1959), 1327.

\section{DePartment of Applied Mathematics}

UNIVERSITY OF LIVERPOOL 